\title{
A FIXED POINT THEOREM AND ITS APPLICATIONS TO A SYSTEM OF VARIATIONAL INEQUALITIES
}

\author{
QAmRul Hasan ANSARI AND Jen-ChiH YAO
}

\begin{abstract}
In this paper, we first prove a fixed point theorem for a family of multivalued maps defined on product spaces. We then apply our result to prove an equilibrium existence theorem for an abstract economy. We also consider a system of variational inequalities and prove the existence of its solutions by using our fixed point theorem.
\end{abstract}

\section{INTRODUCTION}

By using a partition of unity, very recently Lan and Webb [8] have obtained some fixed point theorems for a family of multivalued maps defined on product spaces without compactness assumptions on the domain and range sets. Ding, Kim and Tan [6], Wu [13], Wu and Shen [14] and Yannelis and Prabhakar [15] have also studied such types of fixed point theorems with compactness assumption on the range set, which are well suited to prove equilibrium existence theorems for an abstract economy.

Pang [9] showed that a variety of equilibrium models, for example, the traffic equilibrium problem, the spatial equilibrium problem, the Nash equilibrium problem and the general equilibrium programming problem can be uniformally modelled as a variational inequality defined on the product sets. He decomposed the original variational inequality into a system of variational inequalities which are easy to solve. He also studied the convergence of such methods. The method of decomposition was also used by Zhu and Marcotte [16] to solve a variational inequality problem defined on a set of inequality constraints. By generalising the concept of pseudomonotonicity to the product sets, Bianchi [1] proved the existence of solutions of the system of variational inequalities, that is, a family of variational inequalities defined on a product set.

Inspired by the system of variational inequalities, in the next section we establish a fixed point theorem for a family of multivalued maps defined on product spaces, which generalises the results in $[6,8,14]$ and $[15]$. In Section 3 , we apply our fixed point theorem to prove an equilibrium existence theorem for an abstract economy. In Section 4 , we consider the system of variational inequalities and prove the existence of its solutions by using the result of Section 2 .

Received 28th October, 1998

This research was supported by the National Science Council of the Repubic of China.

Copyright Clearance Centre, Inc. Serial-fee code: 0004-9729/99 \$A2.00+0.00. 
We shall use the following notation and definitions. Let $A$ be a non-empty set. We shall denote by $2^{A}$ the family of all subsets of $A$. If $A$ is a non-empty subset of a topological vector space $X$, we shall denote by $\operatorname{int}_{X}$ the interior of $A$ in $X$. If $A$ is a subset of a vector space, $\operatorname{co}(A)$ denotes the convex hull of $A$.

Let $X$ and $Y$ be two topological vector spaces and $T: X \longrightarrow 2^{Y}$ be a multivalued map. Then $T$ is said to have the local intersection property [14] if for each $x \in X$ with $T(x) \neq \emptyset$, there exists an open neighbourhood $N(x)$ of $x$ such that $\bigcap_{z \in N(x)} T(z) \neq \emptyset$.

The multivalued map $T$ is said to be transfer open-valued [4] if for any $x \in X, y \in$ $T(x)$ there exists an $z \in X$ such that $y \in \operatorname{int}_{Y} T(z)$.

The inverse of $T$, denoted by $T^{-1}$, is the multivalued map from $\mathcal{R}(T)$, the range of $T$, to $X$ defined by

$$
x \in T^{-1}(y) \text { if and only if } y \in T(x) .
$$

\section{FIXED POINT THEOREM}

Let $I$ be an index set and for each $i \in I$, let $E_{i}$ be a Hausdorff topological vector space. Let $\left\{K_{i}\right\}_{i \in I}$ be a family of non-empty convex subsets with each $K_{i}$ in $E_{i}$. Let $K=\prod_{i \in I} K_{i}$ and $E=\prod_{i \in I} E_{i}$.

TheOREM 1. For each $i \in I$, let $S_{i}, T_{i}: K \longrightarrow 2^{K_{i}}$ be two multivalued maps. Assume that the following conditions hold.

(i) For each $i \in I$ and each $x \in K, \operatorname{co}\left(S_{i}(x)\right) \subset T_{i}(x)$ and $S_{i}(x)$ is non-empty.

(ii) For each $i \in I, K=\bigcup\left\{\operatorname{int}_{K} S_{i}^{-1}\left(x_{i}\right): x_{i} \in K_{i}\right\}$.

(iii) If $K$ is not compact, assume that there exist a non-empty compact convex subset $C_{i}$ of $K_{i}$ and a non-empty compact subset $D$ of $K$ such that for each $x \in K \backslash D$ there exists $\tilde{y}_{i} \in C_{i}$ such that $x \in \operatorname{int}_{K} S_{i}^{-1}\left(\widetilde{y}_{i}\right)$.

Then there exists $\bar{x} \in K$ such that $\bar{x} \in T(\bar{x})=\prod_{i \in I} T_{i}(\bar{x})$, that is, $\bar{x}_{i} \in T_{i}(\bar{x})$ for each $i \in I$, where $\bar{x}_{i}$ is the projection of $\bar{x}$ onto $K_{i}$.

Proof: For each $i \in I$, we define a multivalued map $G_{i}: K_{i} \longrightarrow 2^{K}$ by

$$
G_{i}\left(x_{i}\right)=\left\{x \in K: x \notin \operatorname{int}_{K} S_{i}^{-1}\left(x_{i}\right)\right\}=K \backslash \operatorname{int}_{K} S_{i}^{-1}\left(x_{i}\right) .
$$

Then $G_{i}$ satisfies the following conditions:

(a) For each $x_{i} \in K_{i}, G_{i}\left(x_{i}\right)$ is closed in $K$.

(b) For each $i \in I, \bigcap_{x_{i} \in C_{i}} G_{i}\left(x_{i}\right)$ is compact in $K$.

Indeed, if $K$ is compact, $\bigcap_{x_{i} \in C_{i}} G_{i}\left(x_{i}\right)$ is compact since $\bigcap_{x_{i} \in C_{i}} G_{i}\left(x_{i}\right)$ is closed in $K$ by (a). If $K$ is not compact,

$$
\bigcap_{x_{i} \in C_{i}} G_{i}\left(x_{i}\right)=\bigcap_{x_{i} \in C_{i}}\left\{x \in K: x \notin \operatorname{int}_{K} S_{i}^{-1}\left(x_{i}\right)\right\} \subset D \quad \text { by (iii) }
$$


and thus is compact.

(c) Since for each $i \in I, K=\bigcup\left\{\operatorname{int}_{K} S_{i}^{-1}\left(x_{i}\right): x_{i} \in K_{i}\right\}$, we have $\bigcap_{x_{i} \in K_{i}} G_{i}\left(x_{i}\right)=\bigcap_{x_{i} \in K_{i}}\left\{K \backslash \operatorname{int}_{K} S_{i}^{-1}\left(x_{i}\right)\right\}=\emptyset$, for each $i \in I$.

Now, we shall show that there exist $a_{i 1}, \cdots, a_{i i_{i}} \in K_{i}$ such that

$$
\left(\bigcap_{x_{i} \in C_{i}} G_{i}\left(x_{i}\right)\right) \cap\left(\bigcap_{k=1}^{l_{i}} G_{i}\left(a_{i k}\right)\right)=\emptyset .
$$

Suppose that (1) is not true, then for every finite set $\left\{y_{1}, \cdots, y_{n}\right\} \subset K_{i}$, we have

$$
\left(\bigcap_{x_{i} \in C_{i}} G_{i}\left(x_{i}\right)\right) \cap\left(\bigcap_{j=1}^{n} G_{i}\left(y_{j}\right)\right) \neq \emptyset .
$$

Let $P(y)=\left(\bigcap_{x_{i} \in C_{i}} G_{i}\left(x_{i}\right)\right) \cap\left(G_{i}(y)\right)$ for $y \in K_{i}$. Then the family $\left\{P(y): y \in K_{i}\right\}$ has the finite intersection property. Note that $P(y)$ is compact in $K$ for each $y \in K_{i}$ because $\bigcap_{x_{i} \in C_{i}} G_{i}\left(x_{i}\right)$ is compact and $G_{i}(y)$ is closed in $K$. It follows that $\bigcap_{y \in K_{i}} P(y) \neq \emptyset$ and thus $\bigcap_{y \in K_{i}} G_{i}(y) \neq \emptyset$ which is a contradiction to (c).

By (1), we have

$$
\left(\bigcup_{x_{i} \in C_{i}} \operatorname{int}_{K} S_{i}^{-1}\left(x_{i}\right)\right) \cup\left(\bigcup_{k=1}^{l_{i}} \operatorname{int}_{K} S_{i}^{-1}\left(a_{i k}\right)\right)=K .
$$

Let $F_{i}=\operatorname{co}\left(C_{i} \cup\left\{a_{i 1}, \cdots, a_{i l_{i}}\right\}\right)$. Then $F_{i}$ is compact in $K_{i}$. Let $F=\prod_{i \in I} F_{i}$, then $F$ is a compact subset of $K$. By (2), we have

$$
F \subset\left(\bigcup_{x_{i} \in C_{i}} \operatorname{int}_{K} S_{i}^{-1}\left(x_{i}\right)\right) \cup\left(\bigcup_{k=1}^{l_{i}} \operatorname{int}_{K} S_{i}^{-1}\left(a_{i k}\right)\right) .
$$

Since $F$ is compact, there exist $b_{i 1}, \cdots, b_{i t_{i}} \in C_{i}$ such that

$$
F \subset\left(\bigcup_{j=1}^{t_{i}} \operatorname{int}_{K} S_{i}^{-1}\left(b_{i j}\right)\right) \cup\left(\bigcup_{k=1}^{t_{i}} \operatorname{int}_{K} S_{i}^{-1}\left(a_{i k}\right)\right) .
$$

Let $\left\{c_{i 1}, \cdots, c_{i n_{i}}\right\}=\left\{a_{i 1}, \cdots, a_{i l_{i}}, b_{i 1}, \cdots, b_{i t_{i}}\right\}$. We rewrite (4) as follows

$$
F \subset \bigcup_{k=1}^{n_{i}} \operatorname{int}_{K} S_{i}^{-1}\left(c_{i k}\right)
$$

Let $X_{i}=\operatorname{co}\left(\left\{c_{i 1}, \cdots, c_{i n_{i}}\right\}\right)$ and $X=\prod_{i \in I} X_{i}$. We denote by $\Delta_{i}$ the vector subspace of $E_{i}$ generated by $X_{i}$. Then $\Delta_{i}$ is locally convex since it is a finite dimensional subspace. We 
note that $X$ is a compact set in $\prod_{i \in I} \Delta_{i}$, and $X \subset F \subset \bigcup_{k=1}^{n_{i}} \operatorname{int}_{K} S_{i}^{-1}\left(c_{i k}\right)$. Therefore

$$
X \subset\left(\bigcup_{k=1}^{n_{i}} \operatorname{int}_{K} S_{i}^{-1}\left(c_{i k}\right)\right) \cap X \subseteq \bigcup_{k=1}^{n_{i}} \operatorname{int}_{X} S_{i}^{-1}\left(c_{i k}\right) \subset X
$$

and hence $X=\bigcup_{k=1}^{n_{i}} \operatorname{int}_{X} S_{i}^{-1}\left(c_{i k}\right)$

Since $X$ is compact, there exists a partition of unity $\left\{g_{i 1}, \cdots, g_{i n_{i}}\right\}$ subordinated to this finite subcovering such that

(I) for each $k=1, \cdots, n_{i}, g_{i k}: X \longrightarrow[0,1]$ is continuous,

(II) for each $k=1, \cdots, n_{i}, g_{i k}(x)=0$, for $x \notin \operatorname{int}_{X} S_{i}^{-1}\left(c_{i k}\right)$,

(III) for each $x \in X, \sum_{k=1}^{n_{i}} g_{i k}(x)=1$.

For each $i \in I$, we define a map $f_{i}: X \rightarrow X_{i}$ by $f_{i}(x)=\sum_{k=1}^{n_{i}} g_{i k}(x) c_{i k}$, for all $x \in X$. Obviously, for each $i \in I, f_{i}$ is continuous function. For each $x \in X$ and each $k$ with $g_{i k}(x) \neq 0$, we have $x \in \operatorname{int}_{X} S_{i}^{-1}\left(c_{i k}\right) \subset S_{i}^{-1}\left(c_{i k}\right)$ and so that $c_{i k} \in S_{i}(x)$ for each $i \in I$. By (i) and the definition of $f_{i}$, we have for each $i \in I, f_{i}(x) \in \operatorname{co}\left(S_{i}(x)\right) \subset T_{i}(x)$, for all $x \in X$.

Define a map $h: X \longrightarrow X$ by $h(x)=\left(f_{i}(x)\right)_{i \in I}$. Since for each $x \in X, f_{i}(x) \in X_{i}$, it follows that $h$ is well-defined and continuous. By Tychonoff's fixed point theorem [12], $h$ has a fixed point $\bar{x}=\left(\bar{x}_{i}\right)_{i \in I} \in X$. This implies that $\bar{x}_{i}=f_{i}(\bar{x})$ for all $i \in I$. Since for each $i \in I, f_{i}(x) \in T_{i}(x)$ for all $x \in X$, we have $\bar{x}_{i} \in T_{i}(\bar{x})$.

REMARK. (a) Theorem 1 generalises in [6, Theorem 2] and [14], respectively.

(b) The assumtion (ii) in Theorem 1 can be replaced by any one of the following conditions:

(ii)' For each $i \in I, S_{i}^{-1}$ is transfer open-valued.

(ii)" For each $i \in I, S_{i}$ has the local intersection property.

(ii) ${ }^{\prime \prime \prime} \quad$ For each $i \in I$ and $x_{i} \in K_{i}, S_{i}^{-1}\left(x_{i}\right)$ is open in $K$.

Proof: For the proof of (ii)', we refer to [4, Lemma 2.1].

(ii)" Since for each $i \in I, S_{i}(x) \neq \emptyset$, for all $x \in K$, there exists a neighbourhood $N_{i}(x)$ of $x$ such that $\bigcap_{z \in N_{i}(x)} S_{i}(z) \neq \emptyset$. Let $y_{i} \in \bigcap_{z \in N_{i}(x)} S_{i}(z)$, then $y_{i} \in S_{i}(z)$, for all $z \in N_{i}(x)$ and hence $z \in S_{i}^{-1}\left(y_{i}\right)$, for all $z \in N_{i}(x)$. Therefore, $x \in N_{i}(x) \subset S_{i}^{-1}\left(y_{i}\right)$, so that $x \in \operatorname{int}_{K} S_{i}^{-1}\left(y_{i}\right)$. This implies that $K \subset \bigcup\left\{\operatorname{int}_{K} S_{i}^{-1}\left(y_{i}\right): y_{i} \in K_{i}\right\} \subset K$, and hence $K=\bigcup\left\{\operatorname{int}_{K} S_{i}^{-1}\left(y_{i}\right): y_{i} \in K_{i}\right\}$.

(ii)"' From assumption (i) in Theorem 1, we have $K=\bigcup\left\{S_{i}^{-1}\left(y_{i}\right): y_{i} \in K_{i}\right\}$ and since for each $y_{i} \in K_{i}, S_{i}^{-1}\left(y_{i}\right)$ is open in $K$, we get $K=\bigcup\left\{\operatorname{int}_{K} S_{i}^{-1}\left(y_{i}\right): y_{i} \in K_{i}\right\}$. 
When the index set $I$ is a singleton, we have the following result which generalises the well-known Browder fixed point theorem [3], [10, Theorem 1], [14, Corollary 3] and [15, Theorem 3.3].

COROLlary 1. Let $K$ be a non-empty convex subset of a Hausdorff topological vector space $E$, and let $S, T: K \longrightarrow 2^{K}$ be two multivalued maps. Assume that the following conditions hold.

(i) For each $x \in K, \operatorname{co}(S(x)) \subset T(x)$ and $S(x)$ is non-empty.

(ii) $K=\bigcup\left\{\operatorname{int}_{K} S^{-1}(y): y \in K\right\}$.

(iii) If $K$ is not compact, assume that there exist a non-empty compact convex subset $C$ of $K$ and a non-empty compact subset $D$ of $K$ such that for each $x \in K \backslash D$ there exists $\widetilde{y} \in C$ such that $x \in \operatorname{int}_{K} S^{-1}(\widetilde{y})$.

Then there exists $\bar{x} \in K$ such that $\bar{x} \in T(\bar{x})$.

REMARK. The assumption (ii) in Corollary 1 is equivalent to the following condition of Tarafdar [10]:

(ii) $^{\prime}$ For each $y \in K, S^{-1}(y)=\{x \in K: y \in S(x)\}$ contains a relatively open subset $O_{y}$ of $K\left(O_{y}\right.$ could be empty for some $\left.y\right)$ such that $\bigcup_{y \in K} O_{y}=K$.

\section{EQUILIBRIUM EXISTENCE THEOREM}

In this section, we prove an equilibrium existence theorem for a non-compact abstract economy with an infinite number of commodities and an infinite number of agents.

Let $I$ be a (possibly uncountable) set of agents. An abstract economy $\Gamma=$ $\left(K_{i}, A_{i}, B_{i}, P_{i}\right)[6]$, where $A_{i}, B_{i}: K=\prod_{j \in I} K_{j} \longrightarrow 2^{K_{i}}$ are constraint correspondences and $P_{i}: K \longrightarrow 2^{K_{i}}$ is a preference correspondence. An equilibrium [6] for $\Gamma$ is a point $\bar{x} \in K$ such that for each $i \in I$,

$$
\bar{x}_{i} \in B_{i}(\bar{x}) \quad \text { and } \quad A_{i}(\bar{x}) \cap P_{i}(\bar{x})=\emptyset .
$$

When $A_{i}=B_{i}$ for each $i \in I$, these definitions of an abstract economy and an equilibrium coincide with the standard definitions, see for example [2] and [15].

THEOREM 2. Let $\left\{K_{i}\right\}_{i \in I}$ be a family of non-empty convex subsets with each $K_{i}$ in a Hausdorff topological vector space $E_{i}$ and let $\Gamma=\left(K_{i}, A_{i}, B_{i}, P_{i}\right)$ be an abstract economy. Assume that the following conditions hold.

(i) For each $i \in I$ and each $x \in K, \operatorname{co}\left(A_{i}(x)\right) \subset B_{i}(x)$ and $A_{i}(x)$ is non-empty.

(ii) For each $i \in I, K=\bigcup_{y_{i} \in K_{i}} \operatorname{int}_{K}\left[\left\{\left(\operatorname{co} P_{i}\right)^{-1}\left(y_{i}\right) \cup W_{i}\right\} \cap A_{i}^{-1}\left(y_{i}\right)\right]$, where $W_{i}=\left\{x \in K: A_{i}(x) \cap P_{i}(x)=\emptyset\right\}$.

(iii) For each $i \in I$ and each $x \in K, x_{i} \notin \operatorname{co}\left(P_{i}(x)\right)$. 
(iv) If $K$ is not compact, assume that there exist a non-empty compact convex subset $C_{i}$ of $K_{i}$ and a non-empty compact subset $D$ of $K$ such that for each $x \in K \backslash D$ there exists $\widetilde{y}_{i} \in C_{i}$ such that $x \in \operatorname{int}_{K}\left[\left\{\left(\operatorname{co}_{i}\right)^{-1}\left(\widetilde{y}_{i}\right) \cup W_{i}\right\} \cap\right.$ $\left.A_{i}^{-1}\left(\widetilde{y}_{i}\right)\right]$.

Then there exists a point $\bar{x} \in K$ such that $\bar{x}_{i} \in B_{i}(\bar{x})$ and $A_{i}(\bar{x}) \cap P_{i}(\bar{x})=\emptyset$, for all $i \in I$.

Proof: For each $i \in I$, let $V_{i}=\left\{x \in K: A_{i}(x) \cap P_{i}(x) \neq \emptyset\right\}$ and for each $x \in K$, let $I(x)=\left\{i \in I: A_{i}(x) \cap P_{i}(x) \neq \emptyset\right\}$. For each $i \in I$, we define two multivalued maps $S_{i}, T_{i}: K \longrightarrow 2^{K_{i}}$ by

$$
S_{i}(x)= \begin{cases}\operatorname{co} P_{i}(x) \cap A_{i}(x), & \text { for } i \in I(x) \\ A_{i}(x), & \text { for } i \notin I(x)\end{cases}
$$

and

$$
T_{i}(x)= \begin{cases}\operatorname{co} P_{i}(x) \cap B_{i}(x), & \text { for } i \in I(x) \\ B_{i}(x), & \text { for } i \notin I(x)\end{cases}
$$

Then for each $i \in I$ and each $x \in K, \operatorname{co}\left(S_{i}(x)\right) \subset T_{i}(x)$ and $S_{i}(x)$ is non-empty.

Now for each $i \in I$ and $y_{i} \in K_{i}$,

$$
\begin{aligned}
S_{i}^{-1}\left(y_{i}\right) & =\left[\left\{\left(\operatorname{co} P_{i}\right)^{-1}\left(y_{i}\right) \cap A_{i}^{-1}\left(y_{i}\right)\right\} \cap V_{i}\right] \cup\left[A_{i}^{-1}\left(y_{i}\right) \cap W_{i}\right] \\
& =\left[\left(\operatorname{co} P_{i}\right)^{-1}\left(y_{i}\right) \cap A_{i}^{-1}\left(y_{i}\right)\right] \cup\left[A_{i}^{-1}\left(y_{i}\right) \cap W_{i}\right] \\
& =\left[\left(\operatorname{co} P_{i}\right)^{-1}\left(y_{i}\right) \cup W_{i}\right] \cap A_{i}^{-1}\left(y_{i}\right) .
\end{aligned}
$$

From (ii), we have

$$
K=\bigcup_{y_{i} \in K_{i}} \operatorname{int}_{K}\left[\left\{\left(\operatorname{coP}_{i}\right)^{-1}\left(y_{i}\right) \cup W_{i}\right\} \cap A_{i}^{-1}\left(y_{i}\right)\right]=\bigcup_{y_{i} \in K_{i}} \operatorname{int}_{K} S_{i}^{-1}\left(y_{i}\right)
$$

Hence all the conditions of Theorem 1 are satisfied, therefore there exists $\bar{x} \in K$ such that $\bar{x}_{i} \in T_{i}(\bar{x})$ for all $i \in I$. By (iii) and the definition of $T_{i}$, we have $\bar{x}_{i} \in B_{i}(\bar{x})$ and $A_{i}(\bar{x}) \cap P_{i}(\bar{x})=\emptyset$, for all $i \in I$.

\section{SYSTEM OF VARIATIONAL INEQUALITIES}

Let $I$ be an index set and for each $i \in I$, let $E_{i}$ be a Hausdorff topological vector space with its topological dual $E_{i}^{*}$. Let $\left\{K_{i}\right\}_{i \in I}$ be a family of non-empty convex subsets with each $K_{i}$ in $E_{i}$. Let $K=\prod_{i \in I} K_{i}, K^{i}=\prod_{j \neq i, j \in I} K_{j}$ and $E=\prod_{i \in I} E_{i}$. For each $i \in I$, let $A_{i}: K \longrightarrow E_{i}^{*}$ be a given function. Then we consider the system of variational inequalities (in short, SVI) which is to find $\bar{x}=\left(\bar{x}_{i}, \bar{x}^{i}\right) \in K$ such that for each $i \in I$,

$$
\left\langle A_{i}(\bar{x}), y_{i}-\bar{x}_{i}\right\rangle \geqslant 0, \quad \text { for all } y_{i} \in K_{i}
$$


where $\langle\cdot, \cdot\rangle$ denotes the pairing between $E_{i}^{*}$ and $E_{i}$.

The (SVI) was considered by Pang [9] with applications in equilibrium problems. He showed that the traffic equilibrium problem, the spatial equilibrium problem, the Nash equilibrium problem and the general equilibrium programming problem can be modelled as the (SVI). He also investigated the local and global convergence of various iterative methods for solving the (SVI). But he did not discuss the existence of solutions of the (SVI). Later, Bianchi [1] proved the existence of solutions of the (SVI) by generalising the concept of pseudomonotonicity to the product sets and using the Fan-KKM Theorem [7]. The (SVI) was also studied by Cohen and Chaplais [5], and Zhu and Marcotte [16].

First we shall prove an existence theorem for a more general system.

ThEOREM 3. For each $i \in I$, let $f_{i}$ and $g_{i}$ be two real-valued functions defined on $K \times K_{i}$. Assume that the following conditions hold.

(i) For each $i \in I, x \mapsto f_{i}\left(x, y_{i}\right)$ is upper semicontinuous on $K$, for all $y_{i} \in K_{i}$.

(ii) For each $i \in I$ and each finite subset $\left\{y_{i}^{1}, \cdots, y_{i}^{n}\right\}$ of $K_{i}$ we have $f_{i}\left(x, y_{i}^{j}\right)<0$, for all $j=1, \cdots, n$ imply $g_{i}\left(x, \widehat{y}_{i}\right)<0$, where $\widehat{y}_{i}=\sum_{j=1}^{n} \alpha^{j} y_{i}^{j}$, $\alpha^{j} \geqslant 0$ for all $j=1, \cdots, n$ and $\sum_{j=1}^{n} \alpha^{j}=1$.

(iii) For each $i \in I$ and all $x \in K, x_{i} \in K_{i}, g_{i}\left(x, x_{i}\right) \geqslant 0$.

(iv) If $K$ is not compact, assume that there exist a non-empty compact convex subset $C_{i}$ of $K_{i}$ and a non-empty compact subset $D$ of $K$ such that for each $x \in K \backslash D$ there exists $\tilde{y}_{i} \in C_{i}$ such that $f_{i}\left(x, \tilde{y}_{i}\right)<0$.

Then there exists $\bar{x} \in K$ such that for each $i \in I, f_{i}\left(\bar{x}, y_{i}\right) \geqslant 0$, for all $y_{i} \in K_{i}$.

Proof: For each $y_{i} \in K_{i}$, we define a multivalued map $H_{i}: K_{i} \longrightarrow K$ by

$$
H_{i}\left(y_{i}\right)=\left\{x \in K: f_{i}\left(x, y_{i}\right) \geqslant 0\right\} .
$$

Then from (i) we have, for each $i \in I$ and $y_{i} \in K_{i}, H_{i}\left(y_{i}\right)$ is closed in $K$.

Suppose that the conclusion of this theorem is not true. Then for each $x \in K$,

$$
\left\{y_{i} \in K_{i}: f_{i}\left(x, y_{i}\right)<0\right\}=\left\{y_{i} \in K_{i}: x \notin H_{i}\left(y_{i}\right)\right\} \neq \emptyset \text {, for each } i \in J \subseteq I .
$$

Now, we define two multivalued maps $S_{i}, T_{i}: K \longrightarrow 2^{K_{i}}$ by

$$
S_{i}(x)=\left\{y_{i} \in K_{i}: f_{i}\left(x, y_{i}\right)<0\right\} \text { and } T_{i}(x)=\left\{y_{i} \in K_{i}: g_{i}\left(x, y_{i}\right)<0\right\} \text {, respectively. }
$$

Then clearly $S_{i}(x)$ is non-empty, for all $x \in K$ and each $i \in J$.

Let $\left\{y_{i}^{1}, \cdots, y_{i}^{n}\right\}$ be a finite subset of $K_{i}$ such that $f_{i}\left(x, y_{i}^{j}\right)<0$ for all $j=1, \cdots, n$ and $x \in K$. Then from (ii), we have $g_{i}\left(x, \widehat{y}_{i}\right)<0$, where $\widehat{y}_{i}=\sum_{j=1}^{n} \alpha^{j} y_{i}^{j}, \alpha^{j} \geqslant 0$ for all 
$j=1, \cdots, n$ and $\sum_{j=1}^{n} \alpha^{j}=1$. Hence any convex combination of points of $S_{i}(x)$ lies in $T_{i}(x)$. Thus $\operatorname{co}\left(S_{i}(x)\right) \subset T_{i}(x)$, for all $x \in K$ and each $i \in J$.

Since $H_{i}\left(y_{i}\right)$ is closed for all $y_{i} \in K_{i}$ and each $i \in J$, we have $S_{i}^{-1}\left(y_{i}\right)=\{x \in K$ : $\left.f_{i}\left(x, y_{i}\right)<0\right\}=\left[H_{i}\left(y_{i}\right)\right]^{c}$ (the complement of $H_{i}\left(y_{i}\right)$ in $K$ ) is open in $K$ and hence int $_{K} S_{i}^{-1}\left(y_{i}\right)=S_{i}^{-1}\left(y_{i}\right)$ for all $y_{i} \in K_{i}$. Since for each $x \in K, S_{i}(x)$ is non-empty, we have $K=\bigcup_{y_{i} \in K_{i}} S_{i}^{-1}\left(y_{i}\right)=\bigcup_{y_{i} \in K_{i}} \operatorname{int}_{K} S_{i}^{-1}\left(y_{i}\right)$. Then $S_{i}$ and $T_{i}$, for each $i \in J$, satisfy all the conditions of Theorem 1 . Hence there exists $\bar{x} \in K$ such that $\bar{x}_{i} \in T_{i}(\bar{x})$, that is, there exists $\bar{x} \in K$ such that $g_{i}\left(\bar{x}, \bar{x}_{i}\right)<0$ for each $i \in J$,which is a contradiction of assumption (iii). The result is proved.

THEOREM 4. For each $i \in I$, let $f_{i}$ and $g_{i}$ be two real-valued functions defined on $K \times K_{i}$. Assume that the following conditions hold.

(i) For each $i \in I, x \mapsto f_{i}\left(x, y_{i}\right)$ is upper semicontinuous on $K$, for all $y_{i} \in K_{i}$.

(ii) For each $i \in I, f_{i}\left(x, y_{i}\right)<0$ implies $g_{i}\left(x, y_{i}\right)<0$, for all $\left(x, y_{i}\right) \in K \times K_{i}$.

(iii) For each $i \in I$, either $f_{i}$ or $g_{i}$ is quasiconvex in their second variables.

(iv) For each $i \in I$ and all $x \in K, x_{i} \in K_{i}, g_{i}\left(x, x_{i}\right) \geqslant 0$.

(v) If $K$ is not compact, assume that there exist a non-empty compact convex subset $C_{i}$ of $K_{i}$ and a non-empty compact subset $D$ of $K$ such that for each $x \in K \backslash D$ there exists $\tilde{y}_{i} \in C_{i}$ such that $f_{i}\left(x, \tilde{y}_{i}\right)<0$.

Then there exists $\bar{x} \in K$ such that for each $i \in I, f_{i}\left(\bar{x}, y_{i}\right) \geqslant 0$, for all $y_{i} \in K_{i}$.

Proof: The result will follow from Theorem 3 if we show that $\operatorname{co}\left(S_{i}(x)\right) \subset T_{i}(x)$, for all $x \in K$ and each $i \in I$, where $S_{i}$ and $T_{i}$ are defined as in the proof of Theorem 3.

Let $\left\{y_{i}^{1}, \cdots, y_{i}^{n}\right\}$ be a finite subset of $K_{i}$ such that $f_{i}\left(x, y_{i}^{j}\right)<0$, for all $j=1, \cdots, n$. Assume that for each $i \in I, f_{i}$ is quasiconvex in the second variable. Then we have $f_{i}\left(x, \widehat{y}_{i}\right)<0$, where $\widehat{y}_{i}=\sum_{j=1}^{n} \alpha^{j} y_{i}^{j}, \alpha^{j} \geqslant 0$, for all $j=1, \cdots, n$ and $\sum_{j=1}^{n} \alpha^{j}=1$. From (ii), we have $g_{i}\left(x, \widehat{y}_{i}\right)<0$ and hence $\widehat{y}_{i} \in T_{i}(x)$, that is, the convex combinations of points of $S_{i}(x)$ lie in $T_{i}(x)$. Thus $\operatorname{co}\left(S_{i}(x)\right) \subset T_{i}(x)$, for all $x \in K$ and each $i \in I$.

Now let for each $i \in I, g_{i}$ be quasiconvex in the second variable. Since $f_{i}\left(x, y_{i}^{j}\right)<0$, for all $j=1, \cdots, n$, from (ii) we have $g_{i}\left(x, y_{i}^{j}\right)<0$, for all $j=1, \cdots, n$. Since $g_{i}$ is quasiconvex in the second variable, we have $g_{i}\left(x, \widehat{y}_{i}\right)<0$, and hence $\widehat{y}_{i} \in T_{i}(x)$. Thus $\operatorname{co}\left(S_{i}(x)\right) \subset T_{i}(x)$, for all $x \in K$ and each $i \in I$.

REMARK. When $I$ is a singleton, Theorem 3 generalises [11, Lemma 2.1] while Theorem 4 reduces to [11, Lemma 2.1].

Corollary 2. For each $i \in I$, let $A_{i}$ be a function defined on $K$ into $E_{i}^{*}$. Assume that the following conditions hold.

(i) For each $i \in I, A_{i}$ is upper semicontinuous on $K$. 
(ii) There exists a family $\left\{g_{i}\right\}_{i \in I}$ of real-valued functions defined on $K \times K_{i}$ such that

(a) for each $i \in I,\left\langle A_{i}(x), y_{i}-x_{i}\right\rangle<0$ imply $g_{i}\left(x, y_{i}\right)<0$, for all $x \in K$ and $x_{i}, y_{i} \in K_{i}$,

(b) for each $i \in I$ and all $x \in K, x_{i} \in K_{i}, g_{i}\left(x, x_{i}\right) \geqslant 0$.

(iii) If $K$ is not compact, assume that there exist a non-empty compact convex subset $C_{i}$ of $K_{i}$ and a non-empty compact subset $D$ of $K$ such that for each $x \in K \backslash D$ there exists $\tilde{y}_{i} \in C_{i}$ such that $\left\langle A_{i}(x), \tilde{y}_{i}-x_{i}\right\rangle<0$.

Then there exists a solution $\bar{x} \in K$ of the (SVI).

Proof: For each $i \in I$, we define a real-valued function $f_{i}$ on $K \times K_{i}$ by

$$
f_{i}\left(x, y_{i}\right)=\left\langle A_{i}(x), y_{i}-x_{i}\right\rangle, \quad \text { for all }\left(x, y_{i}\right)=\left(\left(x_{i}, x^{i}\right), y_{i}\right) \in K \times K_{i} .
$$

Then by Theorem 4 , there exists $\bar{x} \in K$ such that

$$
\left\langle A_{i}(\bar{x}), y_{i}-\bar{x}_{i}\right\rangle \geqslant 0, \quad \text { for all } y_{i} \in K_{i} .
$$

\section{REFERENCES}

[1] M. Bianchi, Pseudo P-monotone operators and variational inequalities (Universita Cattolica del Sacro Cuore 6, 1993).

[2] A. Borglin and H. Keiding, 'Existence of equilibrium actions and of equilibrium: a note on the 'new' existence theorem', J. Math. Econom. 3 (1976), 313-316.

[3] F.E. Browder, 'The fixed point theory of multivalued mappings in topological vector spaces', Math. Ann. 177 (1968), 283-301.

[4] S.S. Chang, B.S. Lee, X. Wu, Y.J. Cho and G.M. Lee, 'On the generalized quasi-variational inequality problems', J. Math. Anal. Appl. 203 (1996), 686-711.

[5] G. Cohen and F. Chaplais, 'Nested monotony for variational inequalities over product of spaces and convergence of iterative algorithms', J. Optim. Theory Appl. 59 (1988), 360-390.

[6] X.P. Ding, W.K. Kim and K.K. Tan, 'A selection theorem and its applications', Bull. Austral. Math. Soc. 46 (1992), 205-212.

[7] K. Fan, 'A generalization of Tychonoff's fixed point theorem', Math. Ann. 142 (1961), 305-310.

[8] K.Q. Lan and J. Webb, 'New fixed point theorems for a family of mappings and applications to problems on sets with convex sections', Proc. Amer. Math. Soc. 126 (1998), 1127-1132.

[9] J.S. Pang, 'Asymmetric variational inequality problems over product sets: applications and iterative methods', Math. Programming 31 (1985), 206-219.

[10] E. Tarafdar, 'On nonlinear variational inequalities', Proc. Amer. Math. Soc. 67 (1977), 95-98.

[11] E. Tarafdar and G.X.-Z. Yuan, 'Generalized variational inequalities and its applications', Nonlinear Anal. 30 (1997), 4171-4181. 
[12] A. Tychonoff, 'Ein Fixpunktsatz', Math. Ann. 111 (1935), 767-776.

[13] X. Wu, 'A new fixed point theorem and its applications', Proc. Amer. Math. Soc. 125 (1997), 1779-1783.

[14] X. Wu and S. Shen, 'A further generalization of Yannelis-Prabhakar's continuous selection theorem and its applications', J. Math. Anal. Appl. 196 (1996), 61-74.

[15] N.C. Yannelis and N.D. Prabhakar, 'Existence of maximal elements and equilibria in linear topological spaces', J. Math. Econom. 12 (1983), 233-245.

[16] D.L. Zhu and P. Marcotte, 'Co-coercivity and its role in the convergence of iterative schemes for solving variational inequalities', SIAM J. Optim. 6 (1996), 714-726.

Department of Mathematics

Aligarh Muslim University

Aligarh - 202002

India

Present address:

Department of Applied Mathematics

National Sun Yat-sen University

Kaohsiung 804

Taiwan

Republic of China and

Department of Applied Mathematics

National Sun Yat-sen University

Kaohsiung 804

Taiwan

Republic of China 